\title{
Uebersicht der neuesten Leistungen im Umfange der Pharmakologie und beson- ders der medicinischen Botanik
}

$\nabla$ on

Professor Dierbach.

I. Neueste Literatur der speciellen Pharmakologie und ihrer einzelnen Zweige.

- Handbücher der Muteria medica und andere die Araneinittellehrebetreffenden Schriften.

Manuel de Matière medicale, au description abregio des medicamens, avec des tableaux synopliques, montrant les caractères physiques, chimiques et botaniques des principales substances medicamenteuses ou des plantes qui les fournissent; des considerations sur tart de fornuler et lindication de la composition et du mode demploi des principales prèparations officinales des pharmacopoes de Paris, de Londres, dEdinbourg, de Berlin, de Vienne, de St. Petersbourg, par H. Milne Edwards et P. Vauasseur. Jme edition. Paris 1831. ches Crochard.

Macer Floridus de viribus herbarim una cum Walafridi Strabonis, Othonis Cremonensis et Joannis Folsz carminibus similis argumenti, quae secundun codices manuscriptas et veteres editiones recensulit, supplevit, et adnotatione critica instrin. xit Ludovicus Chenlant. Accedit Anonymi Carmen graecum de Herbis, quod e codice Vindobonensi auxit et cum Godofredi Siermanni suisque emerdationibus edidit Julius Sillig. Lipsiae sumptibus Leopoldi Vossii 19.32. Amstclodami apud 1 . Mueller et Comp in do Kalverstraat Kro. 16́3. Londini apud 1. B. Baillère 219 Regent Sireat. Paris apud eundem, rue de lécole de Medicine Nro. 43. bis. $3 \mathrm{fl}$. $9 \mathrm{kr}$. 
A. Westphal. Commentutio inunguralis, doses Medicumentorum dijudicans, detrectatoribus Medicinae breviter refuratis. Marburgi 189g. 4. 16 p.

Die Hausarzneimittel and deren schickliche Anwendung in Hrankheiten, nebst einer genaven Anweisung, aus densel. ben alleriei nütaliche Arzneitn, als PAaster, Salben, Essenzen, Tincturen, Syrupe, Thee u. dgl. za verfertigen, für Aerzte und Fornilienväter in der Stadt und ouf dem Lande; von einem prahtischen Arzte. Neustadt 1832. 8. $54 \mathrm{kr}$.

Podalirius; zwanglose Hefte, als Beiträge zar Hritils der älteren und neueren Arzneimittel, herausgegeben ron P. Philipso n. sites Heft. Nagdeburg 1833. $8 \mathrm{gr}$.

Ueber die Unsicherheit im Gebranche der Arzneien, von sr. Lichtenstädt, Prof. der Medicin in Petersburg.

Hecker literariuche Annalen. Bd. 21. p. -12 . Ein schätzbarer Aufsatz, in welchem zuerst anf die Verschiedenheit der rohen Naturhörper, dann auf die Ungleichr mälsigkeit der Bereitungen aufmerksam genacht wird, und manche zeitgemälse Winke gegeben werden.

Anleitung zur zweckmäfsigen Anwendung der Arzneimittel, welche in die Pharmucopoea militaris Borussica aufgenommen sind, von A. B e y e r, L. preufe. Regimentaraz. Breslan 1832. If. $48 \mathrm{kr}$.

Diese Schrift enthält die preufsischen Militair-Pharmar copoe, die für sich nicht in den Buchhondel tam.

Versuch einer Beantwortong der Frage: $O b$ es nicht an der Zeit sei, den Arzneischatz zu reformiren, und eine namhafte Anzabi von Arzneimittela abzuschaffen. Von dem Regierungs- und Medicinalrath Fischer in Erfurt.

Hufeland Journal. Mïrz $183 \mathrm{a}$. p. 11- 38 .

Ein interensenter Anfrata, der noch gelesen zu werden retdient, obgleich er Vorschläge enthät, die haum je augeführt warden mö.thten. 
Gemeinnuitziger Krankenfreund, Rathgeber und Hausapothelier, enthaltend allgemeine pralitische Vorschriften für die Erhaltung der Gesundheit and diätetisch zwechmäfsiges Verhalten der Kranken urd Wöchnerinnen ron C. A. Wild. Leipzig 283 . I th. $12 \mathrm{gr}$.

b. Pharmakologisehe Choleraschrifton.

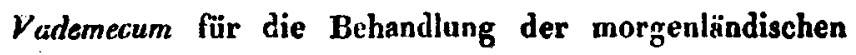
Cholera, oder Materia medica und Handbuch, welches nach den älteren, neueren und neuesten Erlahrungen in Rufsland, Polen, Preufsen, Galizien und Ungarn, für praktiscbe Aerzte bearbeitet worden ist, von D. I. A. Hegar, Churf. Hessischen Hofmedicus etc. Darnsstadt 183ะ. 157 S. 12.

Hausmittel zur Verhütung und Behandlung der Cholera, in Auftrag des Verwaltungssenats der berzogl. Sachsen-Meiningischen Landesregierung zum Druck befördert, von $\mathbf{D}$. E. Hohnbaum, Obermedicinalrath. Meiningen u. Hildburghausen 1832. 8. $32 \mathrm{~S}$.

Die asiatische Cholera, deren Erkennung und Behandung, nebst Angabe der allgemeinen und besonderen Sicherungamittel, für Nichtärzte mit besonderer Rücksicht anf den Bürger und Landmann bearbeitet von Herrm. Schnaubert, Arzt in Cabla. Eisenburg 1831. 8. 2 gr.

Ueber den zweckmäfsigen Gebrauch der Präservative gegen die asiatische Cholera und über die für die erste Anwendung beim Ausbruche derselben im Hause vorräthig $\mathbf{z a}$ haltenden Hülfsmittel, dem grofsen Publikum gewidmet, ron D. H. Messerschmidt, Stadt- und Domphysicus za Naumburg. Naumburg 1831. 8. $4 \mathrm{gr}$.

Des Laien Hausapotheke, zur Beruhigung ror der Cholera - Krankheit. Neuenberg 1831. 2 gr.

Auswahl der besten Mittel und Aecepte gegen die Cholera morbus, gesammelt ans den Schriften der berühmtesten Aerzte aller Nationen. Auch unter dem Titel: Allgemein 
fafsliche Anweisung zur Erkennung, Verbütung und Heilung der asiat. Cholera, nebst Andeutung über die Gefahr dersel. ben in Allgemeinen, zur Belehrung und Beruhigung der Nichtärzte, herausgegeben von einem praktischen Arzte. Leipzig bei Hartmann 1831. 62 S. geh. 4 gr.

Alle rorzüglichst öffentlich gepriesene Mittel gegen die Cholera, gesammelt und heransgegeben zum Gebrauche für Jedermana, von X. Frank. Leipzig 83 ı.

Wird recht gelobt.

Wandtafel zer leichteren Uebersicht der gegen die Cholera bewährtesten Vorsichtsmafsregeln and Hülfsmittel, $\mathbf{z a}$. nächst für seinen praktischen Wirkungshreis entworfen rom Ober-Medicinalrath Dr. Heunemann. Bostock u. Schwerin in der Nillerschen Buchbandlung. Ein Bogen Folio.

Receptirkunst und Formelnbücher.

Vollständiges Recept.'Taschenbuch zur zweckmürsigen Behandlung ailer syphilitischen Kranhheiten. Eine gedrängte Auswahl der besten und neuesten Becepte u. Heilmethoden gegen die sogenannten syphilitischen a. mercuriellen Leiden. Leipzig $1832.21 \mathrm{gr}$.

Formulaire magistral et memorial pharmaceutique par feu Ch. L. Cadet de Gassicourt, $7 e$ edition, par F Cadet de. G. P. L. Colterean et L. Delamortière. Paris 1832. $18 \mathrm{kr}$.

Nouveau formulaire pratique 'des hopitaux et choix des formules des hopitaux civils et militaires de Frances, dingleterre, Iltalie etc. par M. Edwards et P. Vavasseur paris 1839. chez Crochard, place de tècole de Medicine Nir. 13. 1 Vol. petit sa 18.

Pharmacopoeen und sie betreffende Schriften.

Pharmacopoea Slesvico-Holsatica . regia auctoritate edita. Kiliae libraria universitatis 183\%. 4. 6 fl. $18 \mathrm{kr}$.

Supploment to the Pharmucopoeja, and Treatise on Phar. mucology in general, including not only the drugs and prepa. 
retions used iv practitioners of mealicine etc. beirg a complete Dispensalory and bools of formular for consfant reference in medical and veterinary Prsctice efc. By Samuel Frederick Gray. Lecturer on the Materie medica, Pharmaceutical Chemistry and Botany. London 1831. 8. pp. 576. Thomas and George Underwood.

$A$ new Supplement to the Pharmacopejas of London. Bdinburgh, Dublin and Paris; forming a complete Dispensatory and Conspectus; including the new french Medicines and Poisons; with symptoms, treatment and tests, as aell a.s herbs, drugs, compounds, vetcrinary drugs, with the Pharmaiopeja of the Veterinary College, patent medicines, parfumery, peints, varnishes and similars articles, kept in Shops, with theis compositions, imitations, adulterations, and medicinal uses, being a cenerul book of formular and recipes, for daily reference in the laboratory and at the counter. By I. Kinnie Lecturer on Chemistry. London 849. 8. pp.488. Baldwin and Chaddark.

Pharmacopoea of the united states. Philudelphia 1831. 8.

Racolte di farmacopee di N. V. La Ruju. Pisure 1831.

Codex medicamentarius Britanniae sive formulae medicamontorum compositorum, quae in officinis pharmaceuticis Ansliae, Siatiae et Hiberniae prostant. Pars I. Pharmat. Land. Editio tertic. Lipsiae apud Flcischer 1839. I thl.

Pharmocopoca anticholerica extemporeria, exhibens compesitiones medicamentorum a medicini experientissimis ad curam Cholerae asiuticae tam internam quam externam accommodalorum; scripsit F. A. ab Ammon, Prof. in Academia medicochirurgicu Dresdensi et apud Dresdenses medicus et Chirurgus. Lips. apud Voss. 1832. 13/, S. geb. lang fol. I fl. $9 \mathrm{hr}$.

Pharmacopoea anticholericu, cder vollständiger Apparatu: medicamentorım gegen die verschiedenen Hauptformen der Cholera; ein Handbuch für praktische Aerzte a. Chirurgen; enthaltend 283 der bewährtesten anf Autoritäten u. rationtllt 
Heilmethoden gegründete Arzneirorschriften, von Dr. A.P. Wilbelmi. Leipzig bei Hortmann 1831. 215 S. br, mit Umschlag. gr. 12. 54 kr.

Apcthekertaxe, Apothekergewicbt und Apothekenwesen überhaupt.

Apothetertaxe für das Königreich Hannover, die Preise Michaelis 1831 enthaltend. Hannover 1831. 8. Hahn'sche Hofbuchhandlung. $27 \mathrm{kr}$.

Königl. Freufsische Arzneitaxe. Berlin. Verl. von Plohn. 1833. $53 \mathrm{~S}$. 8.

Tafeln der Medicinal-Apothekergewichte aller Länder und freien Städte in Europa in XXVIII Abtheilungen, nach den von hohen Landes-Obermedicinal - Behörden erhaltenen authentischen Angaben entworfen und zum ersten Male auf das Genaueste berechnet von $\mathrm{Fr}$. Lö hman $\mathrm{n}$, Lieutenant von der Armee etc. Leipzig bei Barth 1832. gr. 4. 3 thl. 21 gr.

Medicinisch - pharmaceutisches Wörterbuch.

Stephani Blaneardi Lexicon medicum, in qua artis medicas termini, Anutomiae, Chirurgiae, Pharmacias, Chemias, Rei batanicae, propere dilucide breviterque exponuntur. Editio novissima mullum amendata et aucta a Car. Galtl. Kïhn. Vol. 1. A-1. S maj. Lips. Schwieckert. 4 th. 8 gr.

\section{Medicinisch - pharmaceutische Botanik im Allgemeinen.}

Anleitung zur Erkennung der in der Arzneikunde gobränchlichen phanerogamischen Gewächse, so wie anoh solcher, welche damit verwechselt yerden bönnen, nach den natürlichen Familien, nebst einer kurzen Auseinandersetzung der wichtigsten Pflanzensysteme, einer hürzern alphabetisch geordneten Terminologie ind einem vollatändigen Register aller Namen und Synonyme der darin besehriebenen Gewächse und der davon in arzneiliche Anwendung gezogenen Gegen- 
stände. Für Mediciner und Pharmaceuten von A. B E rg. Berlin 1832. Verlag ron Friedr. Aug. Herber. afl. 48 kr.

Handbuch der medicinischen und Farbekräuter, Blätter, Blüthen, Samen und Wurzeln wilder und lultivirter Pllanzen und Bäume Deutschlands, als Anweisung in welcher Form und welcher Orten dieselben zu erscheinen pflegen, auf welche Weise diesclben anzubinden, und was man beim Einernten und Einsammeln zu beobachten hat. Für Apotheher und Droguisten, vorziigglich aber für Gartenbesitzer, Land - und Waldbewohner als solche, die sich mit dem Anbaue und Einsammeln obiger Produkte beschäftigen, von $C$. A. Schmidt. Gotha und Erfurt. Henningsche Buchhandlung 2833. 8. I fl. $21 \mathrm{kr}$.

Henric. Frideric. Aug. Sasse Lemgovio-Lippiensis. Dissertatio philosophica inauguralis, exhibens observationes quaedam, de plantarum officinalium Collcctione et Praeparatione. Groningae 1828 .

Deutschlands pharmaceutische Flora in getrockneten Exemplaren, oder systematische Aofsteliung der deutschen Pflanzen, welche in der neuen preufsischen Pharmakopöe aufgenommen sind, gesammelt und herausgegeben von $\mathrm{D} \mathrm{a}$ vid Dieterich, Herausgehers des Herburii Florae Germanicue. Ohne Vorrede und andern Text. Jena in Commission bei August Schmidt. 183o. Folio 3 thl.

Ich habe diese Sammlung nicht gesehen. Hornung hat davon eine nicht empfehlende Nachricht gegeben in Heckers literarischen Annalen Bd. 20. p. 352.

2. Gautier. Manuel des plantes medicales, ou description, usage et culture des Vegeteaux indigenes, employés en medicine. Paris 1821. OVol. 18. 1140 S.

The Companion for the kilchen Garden, a History of Vegetubles cultivated in Great Britain, comprising their botanical medicinal edible and chemical Qualitres Natural History 
and relation to art, science and commerce. By Henry Phillips. London 1831. new edition. 2 Vol. in 8.

Grundrils der medicinischen Botanik, als Leitfaden bei Vorlesungen so wie zum Selbstudium und besonders zum repetitorischen Unterricht für Studirende, auch zum Gebrauch für Aerzte urd Pharmaceuten. Nach den natïlichen Familien des Gewächsreichs, mit Angabe der Linneischen Klassification bearbeitct, von Dr. G. W. B is ch off. Heidelberg .83ı. 8.

Zweite Abtheilung. Heidelberg 163s.

Afbeelding der Artzenygewassen, welhe in de Nederlundsthe Apotheek uls Zoodonig vermeld zyn; door N. Anslyk. Nr. I. 1ot. IV. Aflevering. Te Amsterdum by I. C. Sepp en Zoon. 1829 en 30. Fol.

Handbuch der medicinisch - pharmacentischea Botanil. Nach den natürlichen Familien des Gewächsreiches bearbeitet von D. T. F. I. Nees v. Esenbect a. D. C. H. Ebermajer. Illr Theil. Düsseldorf bei Arnz et Comp. 1832. 8.

Grundrifs der Pharmakognosie des Pllanzenreiches, zum Gebrauche bei akademischen Vorlesungen, so wie fir Aerzte, Apotheker und Droguisten entworfen, ron Dr. Theodor Wilhelm Christian Martius, Apotheker in Erlangen und Privaldocenten an der dasigen kön. Unirersität. Erlang. bei Johann Jakob Palm und Ernst Enke. 1832. 8.

Abbildung and Beschreibung aller in der Pharmacopoea borussica aufgetührten Gewächse, herausg. von F. Guimpel, Text von Dr. F. L. v. Schlechtendal. Berlin 1831. 4.

Band 2. Heft 5 und 6 ; sie enthalten nebst dem dazu gehörigen Texte folgende Abbildungen:

Nr. 125. Bactyrilnbium Fistula.

- 126. Anethum graveolens.

- 127. Daucus Carota.

- 128. Coriandrum sativum.

- 18g. Pimpinella Anisum. 
- ABo. Pimpinella Saxifraga.

- 131. Carum Carvio

- 132. Asarum europacum.

- 133. Rubia tinctorum.

- 134. Anchusa tinctoria.

- 135. Iris forentina.

- 136. Sirychnos Nuiv vomica.

Siebentes und achtes Heft:

Nr. 127. Ocimum Basilicum.

- 0.38. Bryonia alba.

- 1.39. Bryonia dioica.

- 140. Vitis vinifera (varietas microcarra).

- 141. Eadem (uarietas mer rocarpa)

- 148. Garcinia Cambogia.

- 143. Melissa officinalis.

- 144. Rubus fruticosus.

- 145. Rubus idaeus.

- 146. Polygala amara.

- 147. Polygala Amarella.

- 148. Liquidambar styracifua.

Der zu diesen beiden Heften gebörige 'Text ist bis jetzt noch nicht geliefert worden.

Fr. Goebels pharmaceutische Waarenkunde mit illuminirten Kupfern. Fortgesetzt ron Dr. Gustav Kunze, aufserordentl. Prof. der Medicin an der Universität Leipzig. Zweiten Bandes fünftes Heft. Eisenach bei Bärecke 183a.

Da die Einrichtung und Anordnung der Gegenstände in diesem schätzbaren Werke bereits früher zurtichend angegeben worden ist, so mag es genügen, den lohalt dieses neueston Heftes blos anzudenten.

Radix Tormentillac. Tormentill -, Ruhr-oder Blutwurzel ron Tormentilla srecta $L$. Tab. XXI. fig. 1 .

Radix Hellebori albi s. Ellebori albi etc. Weifse Nieswurz von Verutrum album and von Veratrum Lobelianum Bernh. T'al. XXI. fig. 2.

Radix Sanguinariae canadensis. Blutwurzel, Hothwurzel, Tarmeric der Indianer, von Sanguinuria casnadensis L. Tab. XXI. fy. 3.

Radix Cyclaminis s. Arthanitae. Erdscheibenworzel, Saubrod von Cgclamen europaeum L. Tab. XXI. f4. 4 .

Radix Artemisiae s. Parthenit. Beifufswurzel, von Artemisia vulgaris L. Tab. XXII. fig. .

Radix Bardanae s, Lappae majoris s. Personotue, Ulletterwurzel von Aictium Burdana Willd, A. majus Gimel. A. minus Schkuhr. Tab. XXII. fig. 2. 
Rudix Iridis sele Irios forcntinae. Florentinische Voilthenwurzel, von lis florentina $L$. u. I. pallida. Lerm. Tab. XXIII. fig. 1.

Radix Iridis nostratis s. Gladioli caerulei. Blaue Schwertel. oder Lilienwurzel von $I$ is germanica $L$. Tab. XXIII. fig. a.

Radix Althaeae s. Bismalvae. Eibischwurel, von Althaea officinalis L. T'al. AXIIl. fig. 3.

Radix Curcumae s. Terra merita. Gilbwurzel. Tab. $X X I V$. fo. et a. C. longa et rotunda, von Curcuma longa $L$

Radix Zedoariae. Zittwerwarzel. Tal. XXlF. fig. 3 at 1. Die lange und runde form von Curcuma Zedoaria Roscae.

hadix $Z_{i n g}$ iberis albi et nigri. Ingwerwurzel ron Zingiber officinale Roscoe. Tab. XXIF. fig. 5.

Badix Arnica es. Doronici germanici. Wohlverleib, von Arnica montana L. T. X.IT. fig. 1.

Radix Caryophyllatae s. Sanamundae. Nelkenwurzel, ron Geum arbanum L. Tazb. XXV. fig. 2.

Radix Gratiolae s. Gratiae Dei. Furpurkrautwurzel, von Gratiola officinalis L. Tab. XXY. fig. 3 .

Radix Serpentariae s. Contruyerwae virginianue. Virginischer Baldrian, von Aristolochia Serpentaria L. Tab. $X X \mathcal{H}$. fig. 4 .

Monographia generis Capsici, auctore A. Fingerhuth medicinae Doctore. 4to. cum tabulis decem coloralis. Dasseldorpii, sumptibus Arnz et Comp.

Durch die folgenden wenigen Zeilen erlaubt sich Beferent die Aufmerksamkeit des botanischen und pharmaceutischen Publicums auf ein Werts zu leiten, dessen Gegenstand die monographische Behandlung einer Pllanzengattung ist, die sowohl in medicinischer als ïhonomischer und botanischer Hinsicht gleich mannigfaltiges Interesse bietet. Die Gattung Capsicum ist $z$ war als eine der natürlicbsten schon large beliannt, aber eben diese nahe Verwandtschaft der Atten unter sich machte die wissensehaftliche Bestimmung der. selben seh: schwierig und nach Blüthen und Blättern unmöglich. Wichtigere Charactere zur Begründung and Feststcllung der Arten suchte der Herr Verfisser daher im lelche und der Frucht und vorzïglich waren es die Form und Oberfläche des Melchs, seine Zälune, hinsichtlich ilser Form und Richtung, die Verinderangen, welche diese Theile nach 